\title{
Autonomous and Dynamic Inter-Cell Interference Coordination Techniques for Future Wireless Networks
}

\author{
Mahdi Ezzaouia*†, Cédric Gueguen ${ }^{\ddagger}$, Mohamad Yassin ${ }^{\S}$, Mahmoud Ammar ${ }^{\dagger}$, \\ Xavier Lagrange*, Ammar Bouallegue ${ }^{\dagger}$ \\ *IMT Atlantique, IRISA, 2 Rue de la Chataigneraie, 35576 Cesson Sévigné, France \\ Email:mahdi.ezzaouia@imt-atlantique.fr \\ ${ }^{\dagger}$ University of Tunis El Manar, National Engineering School of Tunis, \\ Communications Systems Laboratory, 1002 Tunis, Tunisia \\ ${ }^{\ddagger}$ University of Rennes 1, IRISA, Campus de Beaulieu, 35042 Rennes, France \\ ${ }^{\S}$ Orange Labs, 4 Rue du Clos Courtel, 35510 Cesson Sévigné, France
}

\begin{abstract}
Inter-Cell Interference Coordination (ICIC) techniques are proposed as solutions to alleviate the negative impact of interference on system performance, while enhancing the provided Quality of Service (QoS). Typically, the available bandwidth is divided into inner and edge sub-bands. Users are also classified into interior and edge users. The available resources in each zone are exclusively allocated to users belonging to this zone. Mobile users classification is usually based on a threshold that can be either a given mean SINR value or a given distance. However, ICIC approaches based on these static parameters cannot efficiently manage non-homogeneous distribution of users. In this paper, we introduce a dynamic handoff algorithm that aims to adapt static ICIC schemes to uneven distribution of users. Our new solution dynamically computes the classification of active users into interior and edge users, based on a heuristic load balancing algorithm. In our proposal, each cell autonomously reconfigures its bandwidth allocation constraints without modifying bandwidth repartition across the cellular network. This makes the solution well adapted to the non-uniform repartition of users at the multicell scale. Simulation results show that the proposed scheme improves bandwidth usage, reduces packet delay, and increases user satisfaction compared to state-of-the-art ICIC techniques.
\end{abstract}

Keywords-Wireless networks; Resource allocation; Inter-cell interference coordination; Scheduling.

\section{INTRODUCTION}

Future cellular networks should be adapted to the ever increasing demand of mobile broadband communications which will increase at a Compound Annual Growth Rate (CAGR) of 53 percent from 2015 to 2020, reaching 30.6 exabytes per month by 2020 [1]. In this context, the future Fifth Generation $(5 \mathrm{G})$ networks are expected to respond to capacity and throughput challenges by the dense deployment of cells, and the usage of aggressive frequency reuse schemes. However, in order to reach $5 \mathrm{G}$ capacity and throughput goals, we have to deal with Inter-Cell Interference (ICI) problems caused by the usage of the same spectrum in adjacent cells. Indeed, ICI has a negative impact on the provided QoS particularly for cell edge users. In this context, ICIC techniques [2] have been designed in order to reduce interference between the cells, based on the coordination of bandwidth and power allocation.

The Fractional Frequency Reuse (FFR) [3] technique is proposed to protect Cell Edge Users (CEUs) from the negative impact of interference. In each cell, the available bandwidth is divided into two disjoint bands, called center (inner) and edge (outer) bands. On the one hand, in a cluster of $n$ cells, Cell Center Users (CCUs) are served using the inner band with a reuse factor equal to one. On the other hand, CEUs share the edge band with a reuse factor of $n$, that is usually equal to three. Consequently, CEUs of the adjacent cells operate on disjoint spectrum, and FFR eliminates ICI for these users. Figure 1(a) shows a cluster of three adjacent cells, where the FFR scheme is used. Another version of FFR in the time domain, called (FTR) is proposed in [4].

The Soft Frequency Reuse (SFR) [5] technique has been designed to reach performance levels comparable to those of the frequency reuse 1 model, while reducing interference. Unlike the FFR technique, SFR allows the usage of all the available spectrum in each cell. Nevertheless, restrictions are made on the downlink transmission power allocated to the inner sub-band in order to reduce interference for CEUs. In fact, the inner sub-band of a given cell is reused in the edge zones of the neighboring cells. Thus, the inner sub-band used by CCUs is allocated a lower transmission power compared to the edge sub-band [6]. The frequency spectrum allocated to CEUs is used at the maximum downlink transmission power. Indeed, CCUs experience high values of SINR and then, the reduced transmitted power will not have a drastic effect on their QoS. However, CEUs suffer from the lowest values of SINR due to the interferences and the pathloss. In this context, the increased transmitted power will enhance their performances. Figure 1(b) shows a cluster of three adjacent cells where the SFR technique is deployed.

Users classification and bandwidth repartition among regions of the ICIC schemes have a considerable impact on the QoS of cells. These design parameters are discussed in [7]. The authors assume that the resource partitioning between 


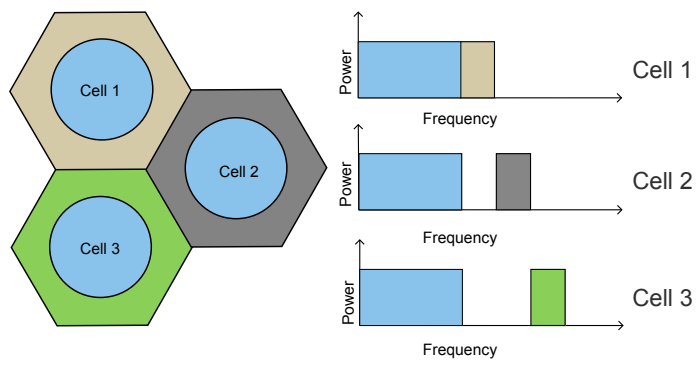

(a) FFR.

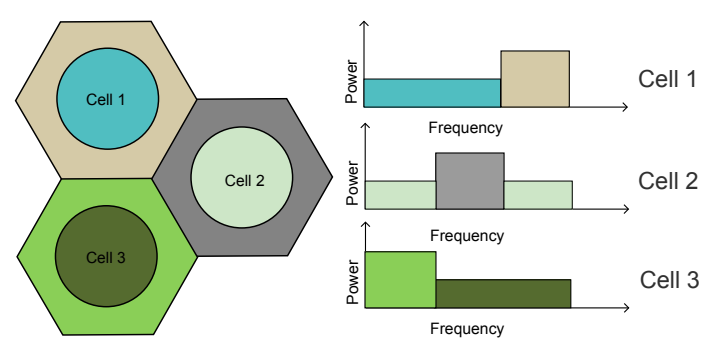

(b) SFR.

Figure 1: The frequency and power arrangements of the spectrum allocation techniques.

CCUs and CEUs is proportional to the square of the ratio of the interior radius $\left(r_{i n t}\right)$ and the cell radius $(R)$. Thus, the total band $N_{\text {band }}$ is divided into two sub-bands: $N_{\text {int }}$ to be allocated to the CCUs, and $N_{\text {ext }}$ reserved for the CEUs. This repartition for the FFR technique is given by:

$$
\begin{gathered}
N_{\text {int }}=\left[N_{\text {band }}\left(\frac{r_{\text {int }}}{R}\right)^{2}\right] . \\
N_{\text {ext }}=\left[\left(N_{\text {band }}-N_{\text {int }}\right) / 3\right] .
\end{gathered}
$$

For the SFR scheme, the bandwidth repartition is given by:

$$
\begin{gathered}
N_{\text {int }}=\left[N_{\text {band }}\left(\frac{r_{\text {int }}}{R}\right)^{2}\right] . \\
N_{\text {ext }}=\min \left(\left[\left(N_{\text {band }}\right) / 3\right], N_{\text {band }}-N_{\text {int }}\right) .
\end{gathered}
$$

In this context, all these parameters have to be configured in advance by the cellular operator. Moreover, this solution is only optimal when the users are uniformly distributed through the network. However, in realistic scenarios, there is no reason for the users to be evenly distributed neither at the scale of a single cell, nor at the level of the entire cellular network.

In [8], the authors introduce a de-centralized dynamic ICIC method where information concerning the cell edge bands are exchanged through the X2 interface. The solution consists in optimizing (shrinking/expanding) the cell edge band based on the interference level. However, each Base Station selects a pre-determined number of frequency resources as cell edge band regardless of cell edge users distribution. Therefore, this solution cannot deal with situations where a large number of users are classified as CEUs since the pre-determined edge sub-band would be insufficient to serve them.

In order to classify the User Equipments (UEs) into cell edge and cell center UEs, three approaches are considered in [9]. The first one consists in employing a distance threshold: if the distance between the base station and the user is smaller than a given threshold, this user will be served using the inner sub-band; otherwise, it will be served using the edge sub-band. The second scheme is similar to the first one, but it uses an SINR threshold instead of the distance. The authors introduce a third scheme based on a load balancing approach called LBA, which takes into account the resource availability of each band and the total available bit rate that could be provided to the users. Simulation results show that the load balancing solution performs better than the two other schemes. However, this scheme is only optimal when the Reuse- 1 and Reuse- 3 bands are not totally used. Indeed, if the system is overloaded, the LBA scheme will make bad decisions when it assigns the users to the different bands.

The authors in [10] propose a centralized dynamic resource allocation. The proposed solution aims to improve the performance of the cell center and cell edge regions by allocating more resources to the cell center users as compared to the fixed FFR scheme. The resource allocation depends on the cell center area of each cell and on the individual demand of each cell. Although the proposed scheme shows better results in comparison with the conventional FFR, it requires the exchange of a large amount of signaling messages due to the presence of the central controller.

In this paper, we introduce a new load balancing algorithm in order to fairly redistribute users between the different regions of the cell and to improve system performance. Our solution consists in computing the mean number of users that can be supported in each zone based on a load balancing scheme. In fact, our proposal aims to help the overloaded cells to absorb traffic congestion and to decrease user dissatisfaction by a more suitable user repartition through the available sub-bands. In addition, our solution is adequate for non homogeneous users distribution between the cells. Indeed, each cell autonomously performs its configuration regardless of the decisions made by the other cells so that no additional signaling messages between the different nodes are required.

The paper is organized as follow: Our system model is described in sections II. In section III, we introduce our load balancing scheme and explain how our proposed algorithm works. Simulation results are discussed in section IV, and the conclusion is given in section $\mathrm{V}$. 


\section{SYSTEM MODEL}

Our system consists of several adjacent hexagonal cells. A Base Station (BS) equipped with an omni-directional antenna is installed in the center of each cell. We consider the Orthogonal Frequency Division Multiplexing (OFDM) transmission technique with $N$ available subcarriers and $K$ users.

In order to model the FFR scheme, a number of subcarriers $N_{\text {center }}$ equal to $N / 2$ is allocated to the inner band and a number $N_{\text {edge }}$ of subcarriers equal to $N / 6$ is allocated to the edge band $[11,12]$. Thus, the transmission power over each of these subcarriers is given by the following equation:

$$
P_{n}=\frac{P_{t}}{N_{\text {center }}+N_{\text {edge }}} .
$$

According to the SFR scheme, all the subcarriers are used. Thus, we consider that $N_{\text {center }}$ is equal to $2 N / 3$ and $N_{\text {edge }}$ is equal to $N / 3$ [13]. However, the allocated power $P_{\text {edge }}$ for the edge band is larger than the power allocated for the center band $P_{\text {center }}$, such that $P_{\text {center }}=\beta P_{\text {edge }} . \beta$ is called the power ratio $(0<\beta<1)$. Finally, the transmission power allocated to the subcarriers of the center and the edge bands are given by:

$$
\begin{gathered}
P_{n, \text { center }}=\frac{\beta P_{t}}{\beta N_{\text {center }}+N_{\text {edge }}} . \\
P_{n, \text { edge }}=\frac{P_{t}}{\beta N_{\text {center }}+N_{\text {edge }}} .
\end{gathered}
$$

The channel gain $G_{k, n}^{i}$ between the serving cell $i$ and user $k$ on subcarrier $n$ is given by this equation :

$$
G_{k, n}^{\mathrm{i}}=h \times 10^{\frac{\chi \sigma}{10}} \times 10^{-P L\left(d_{k, i}\right)},
$$

where $h$ represents the Rayleigh multipath fading which is modeled by an exponential distribution. $\chi_{\sigma}$ is a log-normal shadowing distribution with standard deviation $\sigma$. Finally, $P L\left(d_{k, i}\right)$ is the path loss model given by:

$$
P L\left(d_{k, i}\right)=15.3+37.6 \log _{10}\left(d_{k, i}\right),
$$

where $d_{k, i}$ is the distance (in meters) that separates the user $k$ from its serving cell $i$.

The signal to interference plus noise ratio (SINR) of user $k$ associated to cell $i$ on subcarrier $n$ is given by:

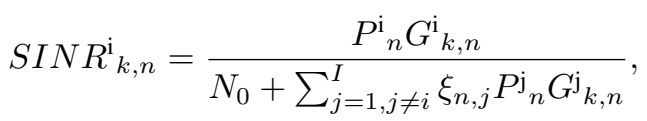

where $P_{n}^{\mathrm{i}}$ and $P^{\mathrm{j}}$ are the transmission power allocated to the subcarrier $n$ by the cell $i$ and by the interfering cell $j$, respectively. Also, $G_{k, n}^{\mathrm{j}}$ is the channel gain between the user $k$ and the interfering cell $j$ on subcarrier $n$, and $N_{0}$ is the thermal noise density. We assume that $\xi_{n, j}=1$ if the interfering cell $j$ is transmitting on the subcarrier $n$; otherwise, $\xi_{n, j}=0$ and there is no collision between the cell $i$ and cell $j$. This parameter distinguishes our work from the state of the art since we are taking into consideration the traffic load of the neighboring cells to compute the SINR formula. Indeed, the more a cell is loaded, the more its bandwidth is used. Consequently, more interference is experienced by its neighboring cells. Moreover, all the works mentioned in the first section use all the subcarriers in order to compute the interference level caused by the neighboring cells since they consider a full buffer traffic model for simulations.

The Adaptive Modulation and Coding (AMC) is used in order to enhance the system's capacity. It consists of the adaptation of the modulation and coding scheme so that it matches the quality of the received signal. We followed the procedure described in [14] to perform the AMC which is inspired from [15]. We start by using Shannon's formula to compute the spectral efficiency $\eta_{k, n}^{\mathrm{i}}$ of user $k$ associated to cell $i$ on subcarrier $n$ as follows:

$$
\eta_{k, n}^{\mathrm{i}}=\log _{2}\left(1+\frac{S I N R_{k, n}^{\mathrm{i}}}{\Gamma}\right)
$$

where $\Gamma$ is a parameter that depends on the target BER and is called $S N R$ gap since it represents the difference between the practical implementations of the MCS and the information-theoretic performances $[15,16]$. In this context: $\Gamma=-\ln (5 B E R) / 1.5$. Finally, we use the efficiency given in Table I to determine the number of bits that could be transmitted to user $k$ over subcarrier $n$.

Table I: LTE CQI index and efficiency.

\begin{tabular}{|l|l|l|l|l|}
\hline $\begin{array}{l}\text { Modulation } \\
\text { Scheme }\end{array}$ & $\begin{array}{l}\text { Approximate } \\
\text { code rate }\end{array}$ & $\begin{array}{l}\text { CQI } \\
\text { Index }\end{array}$ & $\begin{array}{l}\text { Interval for } \\
\eta\end{array}$ & $\begin{array}{l}\text { Efficiency } \\
\text { (bit/symbol) }\end{array}$ \\
\hline No transm & - & 0 & 0 & - \\
\hline & 0.076 & 1 & $0 \div 0.15$ & 0.1523 \\
& 0.12 & 2 & $0.15 \div 0.23$ & 0.2344 \\
QPSK & 0.19 & 3 & $0.23 \div 0.38$ & 0.3770 \\
& 0.3 & 4 & $0.38 \div 0.60$ & 0.6016 \\
& 0.44 & 5 & $0.60 \div 0.88$ & 0.8770 \\
& 0.599 & 6 & $0.88 \div 1.18$ & 1.1758 \\
\hline \multirow{5}{*}{ 16QAM } & 0.37 & 7 & $1.18 \div 1.48$ & 1.4766 \\
& 0.48 & 8 & $1.48 \div 1.91$ & 1.9141 \\
& 0.6 & 9 & $1.91 \div 2.40$ & 2.4063 \\
\hline \multirow{6}{*}{64 QAM } & 0.45 & 10 & $2.40 \div 2.73$ & 2.7305 \\
& 0.55 & 11 & $2.73 \div 3.32$ & 3.3223 \\
& 0.65 & 12 & $3.32 \div 3.90$ & 3.9023 \\
& 0.75 & 13 & $3.90 \div 4.52$ & 4.5234 \\
& 0.85 & 14 & $4.52 \div 5.12$ & 5.1152 \\
& 0.93 & 15 & $\geq 5.12$ & 5.5547 \\
\hline
\end{tabular}




\section{THE PROPOSED SOLUTION}

Our proposal is called Bandwidth Handoff Algorithm (BHA). It consists in switching users from the overloaded sub-band to the lightly loaded one in order to fairly balance the load among the cell center and edge zones. It is designed for the downlink of multiuser OFDMA networks and it does not require any cooperation between the BSs. Thus, our solution only modifies the interior radius of each BS without reconfiguring the initial bandwidth repartition between the cells. This makes it suitable for the non-homogeneous distribution of users at the scale of the cellular network.

In this context, the load balancing scheme can be expressed by:

$$
\frac{K_{c} T_{c}}{N_{c} C_{c}}=\frac{K_{e} T_{e}}{N_{e} C_{e}}
$$

where:

- $K_{c}$ and $K_{e}$ are the mean number of CCUs and CEUs, respectively.

- $T_{c}$ and $T_{e}$ are the instantaneous required throughput of CCUs and CEUs, respectively. Indeed, we assume that the users are characterized by a variable, dynamic and realistic traffic with a high burstiness.

- $N_{c}$ and $N_{e}$ are the number of subcarriers available in the center and the edge sub-bands, respectively.

- $C_{c}$ and $C_{e}$ are the mean number of bits over subcarriers allocated to the two groups of users: CCUs and CEUs, respectively. In fact, we assume that the frequency allocation is performed by the scheduler of each BS (e.g. Proportional Fair, Round Robin, or Best CQI) at each scheduling period.

This load balancing equation aims to optimize the distribution of users through the ICIC sub-bands while absorbing the traffic congestion of the overloaded cells.

Since the number of CCU is equal to $K-K_{e}$ where $\mathrm{K}$ is the total number of users, we can derive the following from eq. (12):

$$
K_{c}=\frac{K T_{e} N_{c} C_{c}}{T_{e} N_{c} C_{c}+T_{c} N_{e} C_{e}} .
$$

Algorithm 1 shows how our proposed solution works. We start by collecting the Channel Quality Indicator (CQI) which is sent by each user as a signaling message. The CQI parameter gives us information about the SINR level of the mobiles in order to sort them in a decreasing order. The required throughput for each group of users is assumed to be known by the eNodeB, and it is updated at each scheduling period. In our model, we choose the Proportional Fair (PF) scheduler in order to allocate the frequency resources to the users. It consists in allocating the frequency resources to a mobile $\mathrm{j}$ when its channel conditions are the most favorable with respect to its time average such as:

$$
j=\operatorname{argmax}_{k}\left(\frac{m_{k, n}}{D_{k}}\right), k=1, \ldots, K,
$$

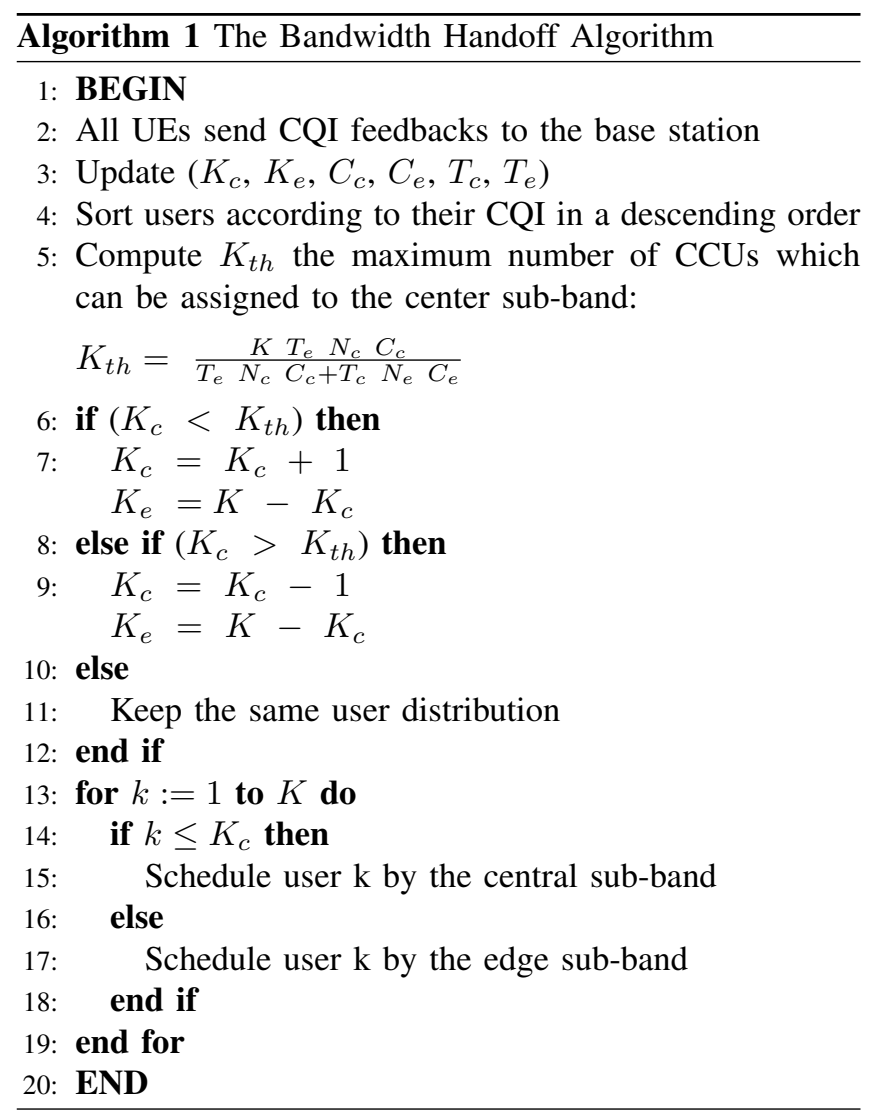

where $m_{k, n}$ is the maximum number of bits that can be transmitted over subcarrier $n$ allocated to the mobile $k$. This value is computed according to our model presented in the previous section. $D_{k}$ is the average throughput provided by the scheduler to user $k$ during the last scheduling periods. The mean number of bits allocated to the two groups of users $\left(C_{c}\right.$ and $\left.C_{e}\right)$ are calculated by the PF scheduler at the previous scheduling period. Also, user repartition $\left(K_{c}\right.$ and $K_{e}$ ) are those computed at the latest processing time. Thus, these parameters are initialized to correct values at the beginning. The BHA computes the mean number of users that can be assigned to the central sub-band based on the load balancing requirement given by eq. (13). However, if our solution ends at this step, an important problem will occur. Indeed, if for example at a scheduling time $t$ we have 10 users considered as CCUs and served by the center sub-band. If at the second scheduling time $\mathrm{t}+1$, the updated number of CCUs to assign to the center sub-band is 18; in order to guarantee a balanced load, 8 users have to be considered as CCUs. In this case, moving in one fell swoop a high number of users between the two sub-bands will cause an instability to our system. In order to avoid this issue, the BHA algorithm moves only one user at each process (line 6 till 11). Finally, the PF scheduler performs resource allocation based on the correct repartition of users among 
the sub-bands.

\section{PERformance EVAluation}

\section{A. Simulation setup and assumptions}

In this section, we evaluate the performance of our proposed BHA solution, and we compare it to the state of the art ICIC techniques. We assume that all UEs run the same videoconference application with an average bit rate equal to $120 \mathrm{Kbps}$. This demanding type of applications produces high bit rate peaks with high burstiness and tight delay constraints. This greatly complicates the task of the schedulers. In addition, each UE has only one service flow with a traffic composed of an MPEG-4 video stream [17] and an AMR voice stream [18].

The interior cell radius that fixes the number of CCUs is one of the most important design parameters of the ICIC techniques. It can be predetermined by the designer of the cellular network based on the provided QoS to the mobiles in each cell zone. In [7], the authors introduce a mathematical-based method in order to determine the subbands repartition. Also, according to this paper, the ICIC scheme reaches its optimal performance in terms of throughput maximization when this interior radius equals 0.65 times the radius of the cell $(\mathrm{R})$. In this work, we assume that the total bandwidth is composed of 75 OFDM subcarriers. By considering this number of subcarriers, this optimal interior radius, and the equations of [7] mentioned in the first section, we determined the size of each sub-band. However, for our BHA solution, we assumed another bandwidth configuration as it was described in our system model. Table II represents the rest of our simulation parameters. Finally, the simulation results were obtained through a discrete events simulator.

\section{B. Performance Metrcis}

1) Packet delay: We assume that UEs' traffic streams are arranged in blocks of bits having the same constant size at the MAC level that we call packets. The packet delay is the time between the appearance of the packet in the transmission buffer and the time of its reception by the user. It corresponds to the packet waiting time in the service flow transmission buffer if the transmission and propagation delays are neglected.

2) Bandwidth usage ratio: The bandwidth usage ratio results are collected through simulations. It is equal to the number of subcarriers allocated by a cell to its own users divided by the total number of its subcarriers.

3) Fairness Index: The fairness index indicates the level of equity of the resource allocation schemes. In this work, we consider the fairness index related to the delay which is given by:

$$
J\left(\bar{R}_{1}, \bar{R}_{2}, \ldots, \bar{R}_{|K|}\right)=\frac{\left(\sum_{k=1}^{|K|} \bar{R}_{k}\right)^{2}}{|K| \cdot \sum_{k=1}^{|K|} \bar{R}_{k}^{2}}
$$

Table II: Simulation parameters.

\begin{tabular}{|l|l|}
\hline Parameters & Value \\
\hline Number of Cells & $\begin{array}{l}10 \text { for the scenario 1 and } \\
7 \text { for the scenario 2 }\end{array}$ \\
\hline Cell Radius (R) & $500 \mathrm{~m}$ \\
\hline Distance threshold & $0.65 R$ \\
\hline $\begin{array}{l}\text { Number of Subcarriers } \\
\text { (OFDMA symbols) }\end{array}$ & 75 \\
\hline SFR power ratio & 0.25 \\
\hline Scheduler & Proportional Fair $(\mathrm{PF})$ \\
\hline BS transmit power & $20 \mathrm{~W}(43 \mathrm{dBm})$ \\
\hline Shadowing & $\begin{array}{l}\text { Log-normal distribution } \\
\left(\mu_{d B}\right.\end{array}$ \\
& $\left.\sigma_{d B}=8 d B\right)$ \\
\hline Multipath fading & Rayleigh fading \\
\hline Target BER & 0.00005 \\
\hline $\begin{array}{l}\text { Thermal noise density } \\
\left(N_{0}\right)\end{array}$ & $-174 \mathrm{dBm} / \mathrm{Hz}$ \\
\hline$P D O R_{\text {target }}$ & $5 \%$ \\
\hline Delay threshold & $100 \mathrm{~ms}$ \\
\hline
\end{tabular}

Where $\mathrm{J}$ rates the fairness of a set of delay values; $|K|$ is the number of users and $\bar{R}_{k}$ is the mean delay of user $k$. This index reaches its maximum value when all the users are characterized by the same delay. Thus, an efficient resource allocation technique reduces the difference in terms of delay between CCUs and CEUs and increases the Jain's fairness index.

4) User dissatisfaction: The users dissatisfaction is based on a delay related metric called Packet Delay Outage Ratio (PDOR) [19] defined as follows: we assume that each mobile application is characterized by a delay threshold noted $T h$. If the packets of a traffic flow experience a delay greater than the delay requirement $T h$, these packets are considered in delay outage. Thus, the PDOR experienced by each service flow is defined as:

$$
P D O R=\frac{n_{\text {out }}}{n_{\text {tot }}}
$$

where $n_{\text {out }}$ is the number of packets in delay outage and $n_{t o t}$ is the total number of transmitted packets. Based on the information provided by the PDOR, we assume that a user $k$ is dissatisfied if its associated service flow experience a PDOR level higher than a given PDOR target $_{\text {: }}$

$$
P D O R_{k}>P D O R_{\text {target }}
$$

\section{Scenario 1: non-homogeneous users distribution among the cells}

In this scenario, we address the problem of the imbalanced users distribution among cells and we underline the benefits of the dynamic interior cell radius configuration by 
comparing our solution to the state of the art ICIC solutions [7].

We simulate two target cells surrounded by 8 neighboring ones. We assume that these two cells have an nonhomogeneous repartition of users between the cell zones: cell 1 contains 10 CCUs and 15 CEUs while cell 2 contains 20 CCUs and only 5 CEUs.

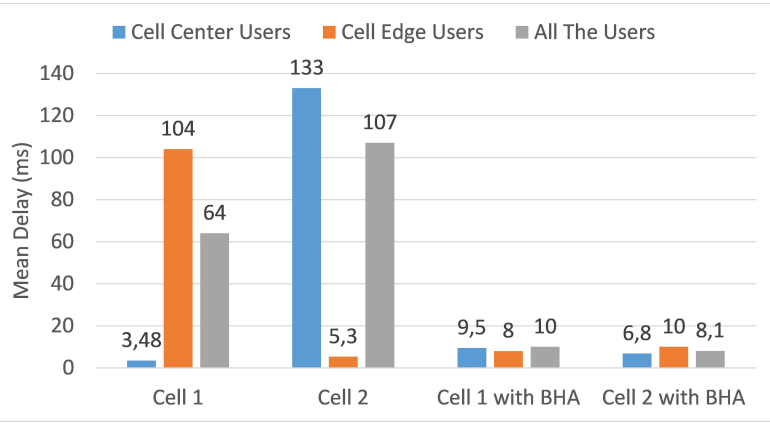

Figure 2: Mean packet delay.

Figure 2 shows the mean delay of the two target cells with the static FFR deployment and with the BHA solution applied to FFR. The results show a significant gap between the CCUs of cell 1 and the CEUs of cell 2. This difference is also observed for the CEUs. Moreover, we notice that cell 1 and cell 2 with the static FFR are characterized by a high delay which exceeds $100 \mathrm{~ms}$. In fact, due to the fixed distance threshold, users are statistically assigned to each band regardless of their distribution between cell zones. Our solution consists in transferring users from the overloaded sub-band to the underloaded one. Consequently, the delay is reduced from $104 \mathrm{~ms}$ to $8 \mathrm{~ms}$ for the CEUs of cell 1 (and from $133 \mathrm{~ms}$ to $6.8 \mathrm{~ms}$ for the CCUs of cell 2). Besides, as more users are assigned by the BHA solution to the center sub-band of cell 1, we observe a slight increase in the delay of CCUs. However, this does not have any impact on the global QoS of the cell since the mean delay of cell 1 is reduced by $54 \mathrm{~ms}$ thanks to our solution.

Figure 3 shows the ratio of radio resources used by the Reuse- 1 sub-band, the Reuse- 3 sub-band and an average for all the cells according to the static FFR and to the BHA solution. As expected, the cell 1 uses only a few amount of its central sub-band and $100 \%$ of its edge sub-band. On the contrary, its neighboring cell uses all its central subband and only $67.4 \%$ of its edge-band. On one hand, our solution maximizes the usage of the radio resources of the under-loaded sub-bands. On the other hand, it delivers a breathing space for users in the overloaded zones since the bandwidth usage ratio is reduced. Consequently, the BHA scheme reduces the bandwidth wastage since it maximizes the usage of the global bandwidth in the two cells.

Results reported in Fig. 4 indicate that the static FFR technique shows a lower fairness index for the two cells.
Indeed, the static scheme is not adapted for situations where a high number of users are classified as CCUs or as CEUs. Consequently, delay discrepancies between users increase. The objective of BHA solution is to decrease the number of users of the edge sub-band of cell 1, and also to decrease the number of users of the center sub-band of cell 2 without any need for cooperation between the cells. Therefore, it shows the highest delay fairness index.

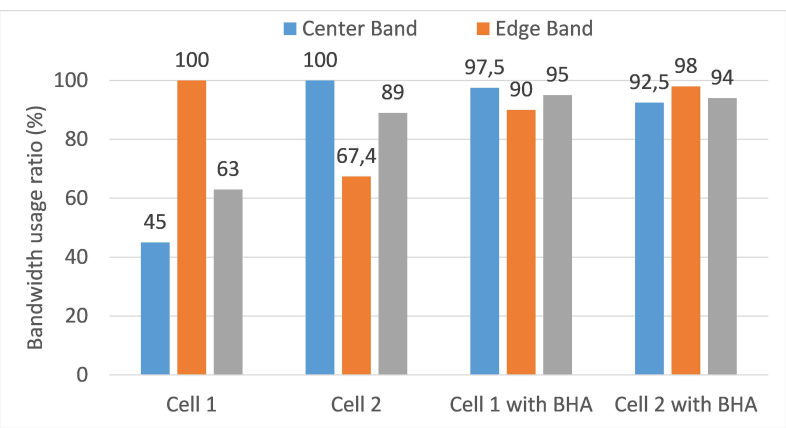

Figure 3: Bandwidth usage ratio.

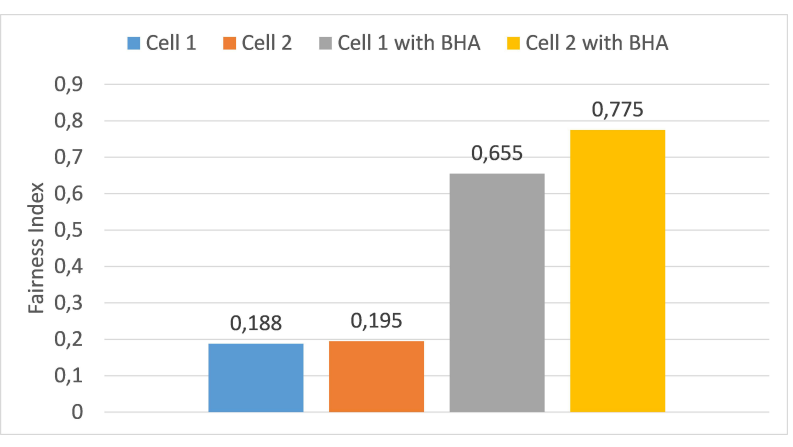

Figure 4: Fairness index.

\section{Scenario 2: cell with different UE distribution}

In this scenario, we consider the impact of user distribution between the cell center and cell edge zones on system performance. We simulate a cluster of seven adjacent hexagonal cells. This cluster contains a central cell with an omni-directional Base station (BS), that also has six neighboring BSs. We focus our study on the center cell that contains 24 UEs. The users are placed uniformly in each cell zone in a manner that the number of CCUs varies between 2 and 22 (between $8.3 \%$ and $91.6 \%$ of the total users). The idea behind this scenario is to study the performance of static ICIC solutions and also the performance of our proposed technique under different levels of non-uniform UEs distribution among the cell zones.

In Fig. 5, we represent the mobile dissatisfaction versus UEs repartition. Results show that our autonomous and 
dynamic BHA solution applied to FFR and SFR is characterized by the lowest percentage of dissatisfaction when the majority of UEs are in the cell-edge zones. Indeed, the dissatisfaction is divided by 2 for the FFR scheme when there are approximately only $10 \%$ of UEs in the center region and is also divided by 3 for the $\mathrm{SFR}_{B H A}$ compared to SFR. When the majority of UEs are in the cell edge region, although they are immune to ICI thanks to the concept of the ICIC technique, the few available frequency resources are not sufficient to serve them. In this case, our solution moves these UEs from the edge overloaded sub-band to the center sub-band as it is shown in Fig. 6 where we represent the number of UEs assigned to the central sub-band. Let's take a look at the left part of the graphic which reflects the case where the majority of users are in the edge region. When the percentage of CCUs is equal to $8.3 \%$, only two users are affected to the central sub-band and 22 UEs to the edge subband according to the two static ICIC techniques. However, thanks to the BHA solution, $10 \mathrm{UEs}$ are considered as CCUs for $\mathrm{SFR}_{B H A}$ and $12 \mathrm{CCUs}$ for $\mathrm{FFR}_{B H A}$ which decreases the dissatisfaction and improves system performance. We also notice that the same process happens when the majority of users are located in the central region since they are moved to the edge sub-band in order to satisfy the load balancing requirements.

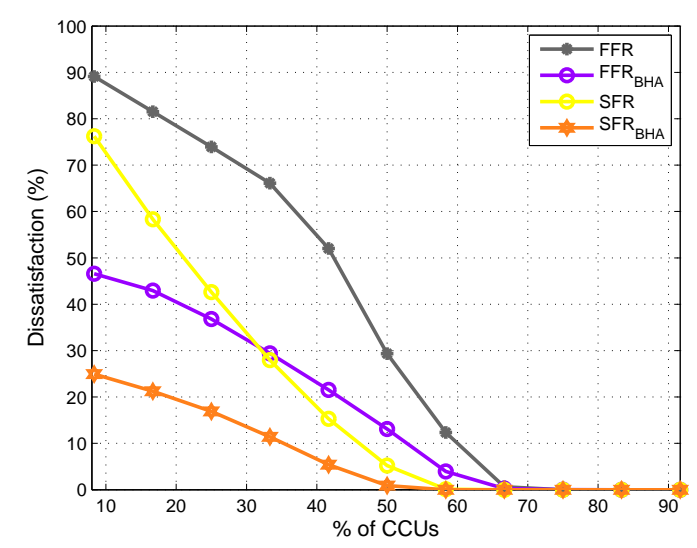

Figure 5: Mean user dissatisfaction in the central cell $P D O R_{\text {target }}=5 \%$.

\section{CONClusion}

In this paper we proposed a dynamic and autonomous solution which can be implemented with the well known FFR and SFR techniques. The proposed method operates locally in each cell without the need for a central controller. It consists in a fair redistribution of the UEs between the ICIC sub-bands thanks to a heuristic load balancing algorithm without changing the bandwidth configuration. Simulation results show that our technique is well adapted to the realistic and non-uniform UEs distribution whether at

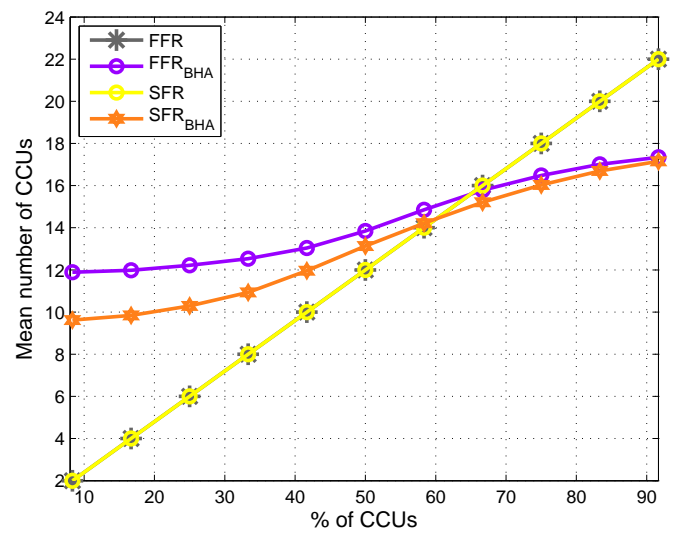

Figure 6: Mean number of users assigned to the center subband.

the multi-cell level or at the single cell level. In addition, our proposed dynamic ICIC technique decreases the wastage of bandwidth, the user's packet delay and user's dissatisfaction compared to the traditional ICIC solutions.

\section{REFERENCES}

[1] Cisco, "Cisco Visual Networking Index: Global Mobile Data Traffic Forecast Update, 2015-2020 White Paper," White Paper, pp. 2 - 3, February 2016.

[2] M. Yassin, M. A. AboulHassan, S. Lahoud, M. Ibrahim, D. Mezher, B. Cousin, and E. A. Sourour, "Survey of icic techniques in lte networks under various mobile environment parameters," Wireless Networks, pp. 1-16, 2015.

[3] S.-E. Elayoubi, O. B. Haddada, and B. Fourestie, "Performance evaluation of frequency planning schemes in ofdmabased networks," IEEE Transactions on Wireless Communications, vol. 7, no. 5, pp. 1623-1633, 2008.

[4] Y. H. Cho, S. Seo, J.-s. Song, S.-H. Lee, and H. S. Lee, "Adaptive fractional time reuse for multi-cell ofdma networks," IEEE Communications Letters, vol. 17, no. 9, pp. 1798-1801, 2013.

[5] X. Yang, "Soft frequency reuse scheme for utran lte," Huawei, 3GPP R1-050507, TSG-RAN1, vol. 41, 2005.

[6] C. Jiming, W. Peng, and Z. Jie, "Adaptive soft frequency reuse scheme for in-building dense femtocell networks," China Communications, vol. 10, no. 1, pp. 44-55, 2013.

[7] T. Novlan, J. G. Andrews, I. Sohn, R. K. Ganti, and A. Ghosh, "Comparison of fractional frequency reuse approaches in the ofdma cellular downlink," in Global Telecommunications Conference (GLOBECOM 2010), 2010 IEEE. IEEE, 2010, pp. 1-5.

[8] D. Kimura, Y. Harada, and H. Seki, "De-centralized dynamic icic using $\mathrm{x} 2$ interfaces for downlink lte systems," in Vehicular Technology Conference (VTC Spring), 2011 IEEE 73rd. IEEE, 2011, pp. 1-5. 
[9] I. N. Stiakogiannakis and D. I. Kaklamani, "Fractional frequency reuse techniques for multi-cellular wimax networks," in Personal Indoor and Mobile Radio Communications (PIMRC), 2010 IEEE 21st International Symposium on. IEEE, 2010, pp. 2432-2437.

[10] M. Hina and S. Sohaib, "Centralized dynamic frequency allocation for cell-edge demand satisfaction in fractional frequency reuse networks," Telecommunication Systems, pp. $1-14,2017$.

[11] M. Yassin, "Inter-cell interference coordination in wireless networks," Ph.D. dissertation, Université de Rennes 1, 2015.

[12] H. Lei, L. Zhang, X. Zhang, and D. Yang, "A novel multi-cell ofdma system structure using fractional frequency reuse," in 2007 IEEE 18th International Symposium on Personal, Indoor and Mobile Radio Communications. IEEE, 2007, pp. 1-5.

[13] M. Assaad, "Optimal fractional frequency reuse (ffr) in multicellular ofdma system," in Vehicular Technology Conference, 2008. VTC 2008-Fall. IEEE 68th. IEEE, 2008, pp. 1-5.

[14] V. Sciancalepore, V. Mancuso, and A. Banchs, "Basics: Scheduling base stations to mitigate interferences in cellular networks," in World of Wireless, Mobile and Multimedia Networks (WoWMoM), 2013 IEEE 14th International Symposium and Workshops on a. IEEE, 2013, pp. 1-9.

[15] G. Piro, N. Baldo, and M. Miozzo, "An lte module for the ns-3 network simulator," in Proceedings of the 4th International ICST Conference on Simulation Tools and Techniques. ICST (Institute for Computer Sciences, Social-Informatics and Telecommunications Engineering), 2011, pp. 415-422.

[16] H. Seo and B. G. Lee, "A proportional-fair power allocation scheme for fair and efficient multiuser ofdm systems," in Global Telecommunications Conference, 2004. GLOBECOM'04. IEEE, vol. 6. IEEE, 2004, pp. 3737-3741.

[17] S. Baey, "Modeling MPEG4 video traffic based on a customization of the DBMAP," in Proc. Int. Symposium on Performance Evaluation of Computer and Telecommunication Systems (SPECTS), San Jose, CA, July 2004, pp. 705 - 714.

[18] P. Brady, "A model for generating on-off speech patterns in two-way conversation," Bell System Technical Journal, vol. 48, 1969.

[19] C. Gueguen, M. Ezzaouia, and M. Yassin, "Inter-Cellular Scheduler for 5G Wireless Networks," Elselvier Physical Communication, vol. 18, no. 2, pp. 113 - 124, 2015. 